\title{
Symbolic computation of degree-three covariants for a binary form
}

\author{
Thomas R. Hagedorn and Glen M. Wilson \\ (Communicated by Scott Chapman)
}

\begin{abstract}
We use elementary linear algebra to explicitly calculate a basis for, and the dimension of, the space of degree-three covariants for a binary form of arbitrary degree. We also give an explicit basis for the subspace of covariants complementary to the space of degree-three reducible covariants.
\end{abstract}

The study of invariant functions was one of the main influences on the development of modern algebra. Consider the following simple example. The group $G=\mathbb{Z}$ acts on $\mathbb{R}$ by addition: $g \cdot x=g+x$. We define a $G$-invariant function to be a real-valued function $f(x)$ on $\mathbb{R}$ such that $f \circ g=f$ for all $g \in G$. In other words, $f(x)=f(g+x)$ for all $g \in \mathbb{Z}, x \in \mathbb{R}$. The invariant functions are precisely the real-valued functions with period one. Hence, geometric information, such as periodicity, can be recovered by studying functions with certain algebraic properties.

In Section 1, we introduce the concepts of an invariant and covariant function for a binary form $Q(x, y)$. The problem of determining the complete set of these functions was widely worked on during the late nineteenth century. Gordan [1868] proved that the ring of invariants (and the ring of covariants) for a degree- $n$ binary form is finitely generated. A milestone in the history of modern algebra was Hilbert's nonconstructive proof [1890] of the following fundamental theorem.

Theorem [Hilbert 1890]. The ring of invariants (and the ring of covariants) for a degree-n homogeneous polynomial in $m$ variables is finitely generated.

Hilbert's theorem says that all invariants (resp. covariants) for a homogeneous polynomial can be expressed as polynomials in a certain finite set of invariants (resp. covariants). Hilbert [1893] subsequently gave a constructive proof of this theorem. The minimal size of the generating set is only known for a few values of $(m, n)$. When $m=2$, this number has been determined for $n \leq 8$ [Bedratyuk 2009; Bedratyuk and Bedratyuk 2008].

MSC2000: 13A50, 15A72, 16W22.

Keywords: theory of covariants, invariant theory, symbolic method, binary forms. 
Let $\mathscr{C}_{d, h}^{n}$ denote the complex vector space of covariants of degree $d$, order $h$ for a degree- $n$ binary form (see Section 1 for a definition). Cayley and Sylvester proved a classical combinatorial formula for $\operatorname{dim} \mathscr{C}_{d, h}^{n}$ [Sturmfels 2008, p. 153]. Algorithms for calculating a basis for $\mathscr{C}_{d, h}^{n}$ are known, but in principle they have only been carried out in a few cases. In general, for a degree- $n$ form in $m$ variables, the most comprehensive treatment is due to Howe [1994], who has given an algorithm for calculating the set of invariants of degree $d \leq 6$.

Here we study the case when $d=3$ and use an elementary argument involving matrix algebra to give an explicit basis for $\mathscr{C}_{3, h}^{n}$ in Theorem 6.1. While our result may not be new, we do not find it in the literature and it corrects the incorrect description of $\mathscr{C}_{3, h}^{n}$ in [Hilbert 1993, p. 62] (see the Historical remark in Section 6). As a corollary, we obtain an explicit form for the Cayley-Sylvester formula in this case. Finally, let $\operatorname{Red}_{3, h}^{n}$ denote the subspace of $\mathscr{C}_{3, h}^{n}$ consisting of reducible covariants (those that are polynomials in lower-degree covariants). In Corollary 6.4, we provide an explicit basis for the subspace in $\mathscr{C}_{3, h}^{n}$ complementary to $\operatorname{Red}_{3, h}^{n}$. Our argument is a variant of the classical straightening algorithm in invariant theory.

In the first two sections of this paper, we define the invariants and covariants of a binary form and review the classical symbolic method. There has been a wealth of excellent introductions to invariant theory recently written [Dolgachev 2003; Kraft and Weyman 1999; Olver 1999; Procesi 2007; Sturmfels 2008] and we refer the reader to them for a more comprehensive introduction to the subject. In the paper's next two sections, we introduce the combinatorial concepts of $\mathscr{H}$-and $\mathscr{U}$-matrices, and establish the relationship with $\mathscr{C}_{3, h}^{n}$. Finally, in Sections 5 and 6, we carry out calculations to determine an explicit basis for $\mathscr{C}_{3, h}^{n}$.

\section{Basic notions}

We review the basic definitions of invariants and covariants found, for example, in [Dolgachev 2003; Kraft and Weyman 1999; Olver 1999]. A binary form $Q(x, y)$ of degree $n$ is a homogeneous polynomial

$$
Q(x, y)=a_{0} x^{n}+\left(\begin{array}{c}
n \\
1
\end{array}\right) a_{1} x^{n-1} y+\ldots+\left(\begin{array}{c}
n \\
n-1
\end{array}\right) a_{n-1} x y^{n-1}+a_{n} y^{n} .
$$

We let $V_{n}$ denote the complex vector space of all binary forms of degree $n$ with complex coefficients. The matrix group $\mathrm{SL}_{2}(\mathbb{C})$ acts on $v \in \mathbb{C}^{2}$ by matrix multiplication $g \cdot v=g v$ and induces an action on

$$
\left(\mathbb{C}^{2}\right)^{*}=\left\{\text { Linear functions } h: \mathbb{C}^{2} \rightarrow \mathbb{C}\right\}
$$

by $(g \cdot h)(v)=h\left(g^{-1} v\right)$. In this context, we regard $x, y$ as the coordinate functions on $\mathbb{C}^{2}$. Thus $x, y \in\left(\mathbb{C}^{2}\right)^{*}$ and $\left(\mathbb{C}^{2}\right)^{*}=\mathbb{C} x \oplus \mathbb{C} y$. If

$$
g=\left(\begin{array}{ll}
a & b \\
c & d
\end{array}\right) \in \mathrm{SL}_{2}(\mathbb{C}),
$$


the explicit action of $g$ on $x, y$ is given by $g \cdot x=d x-b y, g \cdot y=-c x+a y$. Defining $g \cdot\left(x^{a} y^{b}\right)=(g \cdot x)^{a}(g \cdot y)^{b}$, the $\mathrm{SL}_{2}(\mathbb{C})$-action naturally extends to a $\mathrm{SL}_{2}(\mathbb{C})$-action on $V_{n}$. Equivalently, the $\mathrm{SL}_{2}(\mathbb{C})$-action on $\left(\mathbb{C}^{2}\right)^{*}$ extends to the tensor product $\bigotimes_{i=1}^{n}\left(\mathbb{C}^{2}\right)^{*}$, and preserves the subspace $\operatorname{Sym}^{n} \mathbb{C}^{2} \cong V_{n}$.

Remark 1.1. $V_{n}$ is the unique (up to isomorphism) irreducible representation of $\mathrm{SL}_{2}(\mathbb{C})$ of dimension $n$.

Polynomial functions. Invariants and covariants for a binary form of degree $n$ are specific examples of polynomial maps.

Definition 1.2. Let $W=\bigoplus_{i=1}^{k} V_{n_{i}}$. A function $f: W \rightarrow \mathbb{C}$ is a polynomial map of degree $d$ if there is a degree-d homogeneous polynomial $\hat{f} \in \mathbb{C}\left[x_{i j}\right]_{1 \leq i \leq k, 0 \leq j \leq n_{i}}$ such that for all binary forms $Q_{i} \in V_{n_{i}}$, expressed as $Q_{i}(x, y)=\sum_{j=0}^{n_{i}} a_{i j} x^{n-i} y^{i}$ as in (1), we have

$$
f\left(Q_{1}, \ldots, Q_{k}\right)=\hat{f}\left(a_{i j}\right) .
$$

The polynomial $\hat{f}$ is uniquely determined and we identify $f$ with $\hat{f}$. Let $P(W)_{d}$ denote the set of all degree-d polynomial maps on $W$. We say $f$ has multidegree $\mathbf{d}=\left(d_{1}, \ldots, d_{k}\right)$ if

$$
f\left(t_{1} Q_{1}, \ldots, t_{k} Q_{k}\right)=t_{1}^{d_{1}} \ldots t_{k}^{d_{k}} f\left(Q_{1}, \ldots, Q_{k}\right) \quad \text { for all } t_{i} \in \mathbb{C}, Q_{i} \in V_{n_{i}},
$$

and we let $P(W)_{\mathbf{d}}$ denote the set of all such functions.

Example 1.3. Let $k=1, n_{1}=1$, and $\hat{f}\left(x_{0}, x_{1}\right)=x_{0} x_{1}$. Then $f$ defined by $f\left(a_{0} x+\right.$ $\left.a_{1} y\right)=\hat{f}\left(a_{0}, a_{1}\right)=a_{0} a_{1}$ is a polynomial map of degree 2 on $W=V_{1}$. The function $f\left(a_{0} x+a_{1} y\right)=\left|a_{0}\right|$ is not a polynomial map.

More generally, consider a function $f: W \rightarrow V_{h}$. Since $\left\{x^{h}, x^{h-1} y, \ldots, y^{h}\right\}$ is a basis for $V_{h}$, there are functions $f_{i}: W \rightarrow \mathbb{C}$ such that

$$
f=f_{0} x^{h}+f_{1} x^{h-1} y+\cdots+f_{h-1} x y^{h-1}+f_{h} y^{h} .
$$

Definition 1.4. A map $f: W \rightarrow V_{h}$ is a polynomial map of degree $d$ if $f_{i} \in P(W)_{d}$ for each of the functions $f_{i}$ in (2). We let $P(W)_{d, h}$ denote the set of all polynomial maps on $W \rightarrow V_{h}$ of degree $d$. An analogous definition applies if "degree $d$ " is replaced by "multidegree d".

\section{Invariants and covariants.}

Definition 1.5. $A \mathrm{SL}_{2}(\mathbb{C})$-invariant $f: V_{n} \rightarrow \mathbb{C}$ of degree $d$ for a binary form of degree $n$ is a polynomial map $f \in P\left(V_{n}\right)_{d}$ such that

$$
f(g \cdot Q)=f(Q) \quad \text { for all } g \in \mathrm{SL}_{2}(\mathbb{C}) \text { and } Q \in V_{n} .
$$


Example 1.6. The first example of an invariant was discovered by Gauss in his study of binary quadratic forms. Let $Q(x, y)=a_{0} x^{2}+2 a_{1} x y+a_{2} y^{2}$ and define the discriminant $\Delta(Q)=a_{1}^{2}-a_{0} a_{2}$. If $g=\left(\begin{array}{cc}a & b \\ c & d\end{array}\right)$, we have $(g \cdot Q)=b_{0} x^{2}+2 b_{1} x y+b_{2} y^{2}$, where

$$
\begin{aligned}
& b_{0}=a_{0} d^{2}-2 a_{1} c d+a_{2} c^{2}, \\
& b_{1}=-a_{0} b d+a_{1}(a d+b c)-a_{2} a c, \\
& b_{2}=a_{0} b^{2}-2 a_{1} a b+a_{2} a^{2} .
\end{aligned}
$$

$\Delta$ is a degree-two $\mathrm{SL}_{2}(\mathbb{C})$-invariant of a binary quadratic form, as a straightforward calculation shows $\Delta(g \cdot Q)=\Delta(Q)$. It can be shown that if $f$ is a degree- $d$ invariant of a binary quadratic form, then $d$ is even and $f$ is a multiple of $\Delta^{d / 2}$.

Classically, the interest in invariants was to use them to identify geometric properties of projective curves preserved under $\mathrm{SL}_{2}(\mathbb{C})$ transformations. However, invariants are not general enough to specify all such properties. The more general notion of covariants is needed to identify these properties.

Definition 1.7. $A \mathrm{SL}_{2}(\mathbb{C})$-covariant $f$ of degree $d$ and order $h$ for a form of degree $n$ is a polynomial function $f \in P\left(V_{n}\right)_{d, h}$ such that

$$
f(g \cdot Q)=g \cdot f(Q) \quad \text { for all } g \in \mathrm{SL}_{2}(\mathbb{C}), Q \in V_{n} .
$$

Let $\mathscr{C}\left(V_{n}\right)$ denote the vector space of all covariants for a form of degree $n$, and let $\mathscr{C}_{d, h}^{n}=\mathscr{C}\left(V_{n}\right)_{d, h}$ denote the space of those of degree $d$ and order $h$.

Example 1.8. (i) An invariant is a covariant of order $h=0$.

(ii) The simplest example of a covariant is the function $f: V_{n} \rightarrow V_{n}$ of degree 1 , order $n$, defined by $f(Q)=Q . f \in \mathscr{C}_{1, n}^{n}$ is a covariant as the condition $f(g \cdot Q)=g \cdot f(Q)$ is trivially satisfied.

(iii) A more important covariant is the Hessian function. For $Q(x, y) \in V_{n}$, define the Hessian $H: V_{n} \rightarrow V_{2 n-4}$ by

$$
H(Q)=\frac{\partial^{2} Q}{\partial x^{2}} \frac{\partial^{2} Q}{\partial y^{2}}-\left(\frac{\partial^{2} Q}{\partial x \partial y}\right)^{2} .
$$

The Hessian has the property that $H(Q)=0$ precisely when $Q$ is the $n$th power of a linear form. It is a covariant of degree 2 and order $2 n-4$.

More generally, we have the following definition of a covariant. This definition is only used in the next section.

Definition 1.9. Let $W=\bigoplus_{i=1}^{k} V_{n_{i}}$. A covariant of degree $d$, order $h$ for $W$ is a function $f \in P(W)_{d, h}$ satisfying

$$
f(g \cdot Q)=g \cdot f(Q) \quad \text { for all } g \in \mathrm{SL}_{2}(\mathbb{C}) \text { and } Q \in W .
$$


We let $\mathscr{C}(W)$ denote the set of all covariants for $W$. A covariant $f \in \mathscr{C}(W)$ of order $h$ is said to have multidegree $\mathbf{d}$ if $f \in P(W)_{\mathbf{d}, h}$. We let $\mathscr{C}(W)_{d, h}\left(\right.$ resp. $\left.\mathscr{C}(W)_{\mathbf{d}, h}\right)$ denote the sets of covariants in $\mathscr{b}$ of order $h$ and degree $d$ (resp. multidegree $\mathbf{d})$.

\section{The symbolic method}

To prove our results, we will use the classical symbolic method. We review it here. Classically, the legitimacy of the symbolic method was questioned, but it has since been justified [Kung and Rota 1984; Dolgachev 2003]. We now introduce the symbolic method, following the presentation in [Kraft and Weyman 1999] and adopting their notations.

Symbolic method for $\mathscr{C}\left(L^{J}\right)$. Let $J=\{1, \ldots, k\}$. Let $x_{a b}, x_{c}$ for $a, b, c \in J, a \neq b$, denote independent variables and define the polynomial ring

$$
\operatorname{Sym}_{k}=\mathbb{C}\left[x_{a b}, x_{c} \mid a, b, c \in J, a \neq b\right] .
$$

Let $P \in \operatorname{Sym}_{k}$ be a monomial and write

$$
P=\prod_{a, b \in J} x_{a b}^{\lambda_{a b}} \prod_{c \in J} x_{c}^{\sigma_{c}} .
$$

The order ord $P$ and weight wt $P=\left(\mathrm{wt}_{a} P\right)_{a \in J}$ are defined by

$$
\text { ord } P=\sum_{c \in J} \sigma_{c}, \quad \mathrm{wt}_{a} P=\sum_{b \in J}\left(\lambda_{a b}+\lambda_{b a}\right)+\sigma_{a} .
$$

We note that the symmetric group $S_{k}$ naturally acts on $\mathrm{Sym}_{k}$ by

$$
\sigma\left(x_{a b}\right)=x_{\sigma(a) \sigma(b)}, \quad \sigma\left(x_{c}\right)=x_{\sigma(c)} .
$$

Definition 2.1. Let $l_{1}, l_{2} \in L=V_{1}$ and let $l_{i}=a_{i 0} x+a_{i 1} y$. Define

$$
\left[l_{1} l_{2}\right]=\left|\begin{array}{ll}
a_{10} & a_{11} \\
a_{20} & a_{21}
\end{array}\right| .
$$

Let $L^{J}=\bigoplus_{a \in J} L$ and let $l=\left(l_{a}\right) \in L^{J} . L^{J}$ can be identified with $L^{k}$, where $k=|J|$. We denote the maps $l \mapsto\left[l_{a} l_{b}\right]$ and $l \mapsto l_{c}$ by the classical notation $(a b)$ and $c_{x}$, respectively. We have $(a b), c_{x} \in \mathscr{C}\left(L^{J}\right)$.

Theorem 2.2 (First fundamental theorem). The ring $C\left(L^{J}\right)$ is generated by all elements of the forms $(a b)$ and $c_{x}$, for $a, b, c \in J$, and $a \neq b$.

Define the map $\chi: \operatorname{Sym}_{k} \rightarrow \mathscr{C}\left(L^{J}\right)$ by

$$
\chi\left(x_{a b}\right)=(a b), \quad \chi\left(x_{c}\right)=c_{x},
$$

and extend it in the natural way to monomials and all of $\operatorname{Sym}_{k}$. 
Corollary 2.3. $\chi: \operatorname{Sym}_{k} \rightarrow \mathscr{C}\left(L^{J}\right)$ is a surjective homomorphism of algebras. $\chi$ sends a monomial $P$ of order $h$ and weight $w$ to a covariant $\chi(P)$ of order $h$ and multidegree $w$.

Let $\mathfrak{a}=\operatorname{ker} \chi$. Then

$$
\operatorname{Sym}_{k} / \mathfrak{a} \cong \mathscr{C}\left(L^{J}\right) .
$$

We now describe the elements of $\mathfrak{a}$. For all distinct $a, b, c, d \in J$, we have

$$
\begin{gathered}
(a b)+(b a)=0, \quad(a b) c_{x}+(c a) b_{x}+(b c) a_{x}=0, \\
(a b)(c d)+(a d)(b c)-(a c)(b d)=0 .
\end{gathered}
$$

Traditionally, these equations are called syzygies of the first, second, and third type. These syzygies motivate the definition of three subideals of $\mathfrak{a}$.

$$
\begin{aligned}
& \mathfrak{a}_{1}=\left\langle x_{a b}+x_{b a} \mid a \neq b \in J\right\rangle, \\
& \left.\mathfrak{a}_{2}=\left\langle x_{a b} x_{c}+x_{c a} x_{b}+x_{b c} x_{a}\right| \text { distinct } a, b, c \in J\right\rangle, \\
& \left.\mathfrak{a}_{3}=\left\langle x_{a b} x_{c d}+x_{a d} x_{b c}-x_{a c} x_{b d}\right| \text { distinct } a, b, c, d \in J\right\rangle .
\end{aligned}
$$

The second fundamental theorem (or invariant theorem) for $\mathrm{SL}_{2}$ states that these three syzygies generate all the relationships among the covariants $\mathscr{C}\left(L^{J}\right)$.

Theorem 2.4 (Second fundamental theorem). Let $\mathfrak{a}=\operatorname{ker} \chi$. Then $\mathfrak{a}=\mathfrak{a}_{1}+\mathfrak{a}_{2}+\mathfrak{a}_{3}$.

Notation. (i) $\mathrm{Sym}_{k}$ is bigraded by weight and order. Let $\operatorname{Sym}_{k, w, h} \subset \mathrm{Sym}_{k}$ be those elements with weight $w$ and order $h$. Then $\operatorname{Sym}_{k}=\bigoplus_{w, h} \operatorname{Sym}_{k, w, h}$. The ideal $\mathfrak{a}$ is also bigraded and we let $\mathfrak{a}_{w, h}=\mathfrak{a} \cap \operatorname{Sym}_{k, w, h}$.

(ii) When the weight is $w=(n, \ldots, n)$, we write $w=(n)^{k}$ as shorthand.

Classical symbolic description of $\mathscr{C}_{\boldsymbol{k}, \boldsymbol{h}}^{\boldsymbol{n}}$. Equation (3) describes $\mathscr{C}\left(L^{J}\right)$ in terms of the symbolic algebra. Classically, these same symbols were also used to denote covariants in $\mathscr{C}_{k, h}^{n}\left(=\mathscr{C}\left(V_{n}\right)_{k, h}\right)$. We now present this alternate symbolic method and relate the two notations.

Let $Q(x, y)$ be a degree $n$ binary form with coefficients $a_{i}$ as in (1). For each $a \in J$, let $\alpha_{a 0}, \alpha_{a 1}$ be indeterminates with the property that $\alpha_{a 0}^{n-i} \alpha_{a 1}^{i}=a_{i}$, for $i=0, \ldots, n$. Let $a_{x}=\alpha_{a 0} x+\alpha_{a 1} y$ and define

$$
(a b)=\left|\begin{array}{cc}
\alpha_{a 0} & \alpha_{a 1} \\
\alpha_{b 0} & \alpha_{b 1}
\end{array}\right|=\alpha_{a 0} \alpha_{b 1}-\alpha_{a 1} \alpha_{b 0}
$$

Using these definitions, for a monomial $P=\prod_{a \neq b} x_{a b}^{\lambda_{a b}} \prod_{c} x_{c}^{\sigma_{c}} \in \operatorname{Sym}_{k,(n)^{k}, h}$, the expression

$$
\psi(P)=\prod_{a \neq b}(a b)^{\lambda_{a b}} \prod_{c} c_{x}^{\sigma_{c}}
$$


is a well-defined degree- $h$ binary form whose coefficients are homogeneous degree $k$ polynomials in the $a_{i}$. Moreover, the function $Q \mapsto \psi(P)$ can be seen to be a covariant in $\mathscr{C}_{k, h}^{n}$. Let $\psi(P)$ denote this covariant. We note that $\chi(P) \in \mathscr{C}\left(L^{J}\right)$ and $\psi(P) \in \mathscr{C}_{k, h}^{n}$ are different types of covariants, but the symbolic representation of $\chi(P)$ from the previous subsection equals the symbolic representation $\psi(P)$.

Example 2.5. Let $P=x_{a b}^{2} \in \operatorname{Sym}_{2,(2)^{2}, 0}$. In the notation just introduced, the symbol $(a b)^{2}$ for the binary quadratic form $Q$ of (1) represents

$$
(a b)^{2}=\left(\alpha_{a 0} \alpha_{b 1}-\alpha_{a 1} \alpha_{b 0}\right)^{2}=\alpha_{a 0}^{2} \alpha_{b 1}^{2}-2 \alpha_{a 0} \alpha_{a 1} \alpha_{b 0} \alpha_{b 1}+\alpha_{a 1}^{2} \alpha_{b 0}^{2}=2\left(a_{0} a_{2}-a_{1}^{2}\right) .
$$

Thus $\psi(P)$ is the covariant $Q \mapsto 2\left(a_{0} a_{2}-a_{1}^{2}\right)$. Its symbolic representation $(a b)^{2}$ is the same as that of $\chi(P)=(a b)^{2} \in \mathscr{C}\left(L^{2}\right)$.

The two symbolic methods are linked by the following proposition.

Proposition 2.6 [Kraft and Weyman 1999]. There is a surjective homomorphism of vector spaces

$$
\Lambda: \mathscr{C}\left(L^{J}\right)_{(n)^{k}, h} \rightarrow \mathscr{C}_{k, h}^{n}
$$

such that the composition $\Lambda \circ \chi: \operatorname{Sym}_{k,(n)^{k}, h} \rightarrow \mathscr{C}_{k, h}^{n}$ is surjective with kernel

$$
I=\mathfrak{a}_{(n)^{k}, h}+\left\langle P-\sigma \cdot P: P \in \operatorname{Sym}_{k,(n)^{k}, h}\right\rangle .
$$

If $P \in \operatorname{Sym}_{k,(n)^{k}, h}$ is a monomial, then $\Lambda(\chi(P))=\psi(P)$. Moreover, $\Lambda$ sends a symbolic covariant in $\mathscr{C}\left(L^{J}\right)$ to the covariant in $\mathscr{C}_{k, h}^{n}$ with the same symbolic representation.

Corollary 2.7. The map $P \mapsto \psi(P)$ induces an isomorphism $\operatorname{Sym}_{k,(n)^{k}, h} / I \cong \mathscr{C}_{k, h}^{n}$.

This result enables the easy classification of covariants with small degree.

Example 2.8 (Covariants of degree 1). Let $J=\{a\}$. By the corollary, $\mathscr{C}_{1, h}^{n}=0$ when $h \neq n$. When $h=n, \mathscr{C}_{1, n}^{n}$ is generated by $\psi\left(x_{a}^{n}\right)=a_{x}^{n}$. As $I=\mathfrak{a}_{(n)^{1}, n}=0$, $\mathscr{C}_{1, n}^{n}$ is the one-dimensional space generated by $g=a_{x}^{n} \cdot g$ is the trivial covariant with the property $g(Q)=Q$ for any degree $n$ binary form $Q$.

Example 2.9 (Covariants of degree 2). Let $J=\{a, b\}$. We will show that

$$
\operatorname{dim} \mathscr{C}_{2, h}^{n}= \begin{cases}1 & \text { if } h \text { is even, } h \leq 2 n, \text { and } h \equiv 2 n \quad \bmod 4 \\ 0 & \text { otherwise. }\end{cases}
$$

In the former case, $\mathscr{C}_{2, h}^{n}$ is generated by $g=(a b)^{n-h / 2} a_{x}^{h / 2} b_{x}^{h / 2}$. When $h=2 n$, this is the covariant $g$ such that $g(Q)=Q^{2}$. By the corollary, the vector space $\mathscr{C}_{2, h}^{n}$ is generated by the images $\psi(m)$ of the monomials $m=x_{12}^{a} x_{21}^{b} x_{1}^{c} x_{2}^{h-c}$. Since $x_{21}^{b}-(-1)^{b} x_{12}^{b} \in \mathfrak{a}$, we only need consider $m=x_{12}^{a} x_{1}^{c} x_{2}^{h-c}$. Since $m$ has weight $(n)^{2}, a+c=n=a+(h-c)$ and $h=2 c$. Thus if $h$ is odd, $\mathscr{C}_{2, h}^{n}=\{0\}$. If $h$ is even, let $h=2 c$. Then $\mathscr{C}_{2, h}^{n}$ is at most a one-dimensional space generated by the 
image of $m=x_{12}^{n-c} x_{1}^{c} x_{2}^{c}$. For this space to be nontrivial, we must also have $c \leq n$. If $\sigma=(12), m-\sigma(m)=\left(x_{12}^{n-c}-x_{21}^{n-c}\right) x_{1}^{c} x_{2}^{c} \in I$. Substituting for $x_{21}$,

$$
\left(1-(-1)^{n-c}\right) x_{12}^{n-c} x_{1}^{c} x_{2}^{c} \in I .
$$

Hence, when $n-c$ is odd, $m \in I$ and $\psi(m)=0$. Finally, we show that $m \notin I$ when $h=2 c, h \leq 2 n$ and $n-c$ is even. Suppose that $m \in I$. Then the definition of $I$ shows that

$$
x_{12}^{n-c} x_{1}^{c} x_{2}^{c}=\left(x_{12}+x_{21}\right) f+[P-\sigma(P)],
$$

where $f \in \mathrm{Sym}_{2}, \sigma=(12)$, and we can assume $P$ is a linear combination of monomials of the form $x_{12}^{i} x_{21}^{n-c-i} x_{1}^{c} x_{2}^{c}$. Then

$$
P-\sigma(P)=x_{1}^{c} x_{2}^{c} \sum_{i} a_{i}\left(x_{12} x_{21}\right)^{i}\left[x_{21}^{n-c-2 i}-x_{12}^{n-c-2 i}\right] .
$$

Now (5) is an identity in $\operatorname{Sym}_{2}$, so letting $x_{21}=-x_{12}$, we obtain an identity in $\mathbb{C}\left[x_{1}, x_{2}, x_{12}\right]$. But since $n-c$ is even, we obtain $x_{12}^{n-c} x_{1}^{c} x_{2}^{c}=0$, in $\mathrm{Sym}_{2}$, which gives a contradiction. Hence $m \notin I$ and $\mathscr{C}_{2, h}^{n}$ is one-dimensional in this case.

We now define the map Observe that the proof of Theorem 6.1 uses only the statements of Proposition 2.6 and Corollary 2.7. Consider $f \in \mathscr{C}\left(L^{J}\right)_{(n)^{k}, h}$. By Theorem 2.2, $f=\sum_{P} c_{P} P$, where

$$
P=\prod_{a \neq b}(a b)^{\lambda_{a b}} \prod_{c \in J} c_{x}^{\sigma_{c}},
$$

and wt $P=n$, ord $P=h$. We will define $\Lambda(P)$ for each monomial $P$. Then we can extend the domain of $\Lambda$ to all of $\mathscr{C}\left(L^{J}\right)_{(n)^{k}, h}$ by defining

$$
\Lambda(f)=\sum_{P} c_{P} \Lambda(P) .
$$

It remains to define $\Lambda(P) \in \mathscr{C}_{k, h}^{n}$. We will do so by defining $\Lambda(P)(g) \in V_{h}$, for $g \in V_{n}$. Among the many possibilities, fix a choice of integers $\lambda_{a_{i} b_{j}}, \sigma_{c_{l}} \in\{0,1\}$, for $a, b, c \in J, i, j, l \in\{1, \ldots, n\}$ such that

$$
\sum_{i} \lambda_{a_{i} b_{j}}+\sigma_{b_{j}}=1, \quad \sum_{j} \lambda_{a_{i} b_{j}}+\sigma_{a_{i}}=1, \quad \sum_{i, j} \lambda_{a_{i} b_{j}}=\lambda_{a b}, \quad \sum_{l} \sigma_{c_{l}}=\sigma_{c} .
$$

Since $g$ is a complex polynomial of degree $n$ in two variables, it factors as $g=$ $g_{1} \ldots g_{n}$. Define

$$
\Lambda(P)(g)=\frac{1}{(n !)^{k}} \sum_{\substack{\left(\tau_{a}\right)_{a \in J} \\ \tau_{a} \in S_{n}}}\left(\prod_{\substack{a \neq b \in J \\ 1 \leq i, j \leq n}}\left[g_{\tau_{a}(i)} g_{\tau_{b}(j)}\right]^{\lambda_{a_{i} b_{j}}} \prod_{\substack{c \in J \\ 1 \leq l \leq n}} g_{\tau_{c}(l)}^{\sigma_{c_{l}}}\right) .
$$


Because of the summation over $\tau_{a} \in S_{n}, \Lambda(P)(g)$ is independent of the choices of $\lambda_{a_{i} b_{j}}, \sigma_{c_{l}}$, and $g_{i}$. It is clear that $\Lambda(P) \in P\left(V_{n}\right)_{k, h}$. More work shows $\Lambda(P) \in \mathscr{C}_{k, h}^{n}$.

Example 2.10. We illustrate the map $\Lambda$. Consider $P=(a b)^{2} \in \mathscr{C}\left(L^{2}\right)$. We have $k=2$ and ord $P=0$. Then $\lambda_{a b}=2$ is the only nonzero exponent among the $\lambda_{c d}, \sigma_{c}$. Choose $\lambda_{a_{1} b_{1}}=\lambda_{a_{2} b_{2}}=1$ as the only nonzero exponents in (6). Let $g=a_{0} x^{2}+2 a_{1} x y+a_{2} y^{2}=a_{0}\left(x-\alpha_{1} y\right)\left(x-\alpha_{2} y\right)$ and let $g_{1}=a_{0}\left(x-\alpha_{1} y\right)$, $g_{2}=x-\alpha_{2} y$. Then

$$
\begin{aligned}
\Lambda(P)(g) & =\frac{1}{4}\left(\left[\begin{array}{ll}
g_{1} & g_{1}
\end{array}\right]\left[\begin{array}{ll}
g_{2} & g_{2}
\end{array}\right]+\left[\begin{array}{ll}
g_{1} & g_{2}
\end{array}\right]\left[g_{2} g_{1}\right]+\left[g_{2} g_{1}\right]\left[\begin{array}{ll}
g_{1} & g_{2}
\end{array}\right]+\left[\begin{array}{ll}
g_{2} & g_{2}
\end{array}\right]\left[g_{1} g_{1}\right]\right) \\
& =\frac{1}{4}\left(\left[g_{1} g_{2}\right]\left[g_{2} g_{1}\right]+\left[g_{2} g_{1}\right]\left[g_{1} g_{2}\right]\right)=-\frac{1}{2} a_{0}^{2}\left(\alpha_{1}-\alpha_{2}\right)^{2} \\
& =2\left(a_{0} a_{2}-a_{1}^{2}\right),
\end{aligned}
$$

and $\Lambda(P) \in \mathscr{C}_{2,0}^{2}$. This calculation also shows that $\Lambda\left(\chi\left(x_{a b}^{2}\right)\right)=\psi\left(x_{a b}^{2}\right)$ by Example 2.5, which illustrates the second half of Proposition 2.6.

\section{3. $\mathscr{H}$-matrices and $U$-matrices}

In the previous section, we used Corollary 2.7 to classify the covariants of degrees one and two by computing $\mathscr{C}_{k, h}^{n} \cong \operatorname{Sym}_{k,(n)^{k}, h} / I$. For larger degrees, the combinatorics in analyzing $I$ are more difficult. In this section, we introduce $\mathscr{H}$-matrices and $U$-matrices to simplify the analysis and then use them in the next section to classify the covariants of degree 3 . Our goal is to define easily computed maps

$$
\operatorname{Sym}_{3,(n)^{3}, h} \stackrel{\theta}{\rightarrow} \mathbf{H}_{3, h}^{n} \stackrel{\Delta}{\rightarrow} \mathbf{U}_{3, h}^{n},
$$

with $\operatorname{ker}(\Delta \circ \theta)=I$. Then we will be able to explicitly compute $\mathscr{C}_{3, h}^{n}$. He-matrices. To a monomial $P=\prod_{a \neq b} x_{a b}^{\lambda_{a b}} \prod_{c \in J} x_{c}^{\sigma_{c}}$ in $\mathrm{Sym}_{k}$ we associate a $k \times k$
integral matrix $\theta(P)=\left(\theta_{i j}\right)$ by

$$
\theta_{i j}= \begin{cases}\lambda_{i j} & \text { if } i \neq j \\ \sigma_{i} & \text { if } i=j\end{cases}
$$

When $P \in \operatorname{Sym}_{k,(n)^{k}, h}, \theta(P)$ will be an $\mathscr{H}$-matrix of type $(n, k, h)$.

Definition 3.1. Fix integers $k, n>0, h \geq 0$. A $k \times k$-matrix $B=\left(b_{i j}\right)$ is an $\mathscr{H}$ matrix of type $(n, k, h)$ if the coefficients $b_{i j}$ are nonnegative integers satisfying $\sum_{j=1}^{k} b_{j j}=h$ and for each $i$,

$$
\sum_{j=1}^{k} b_{i j}+\sum_{j=1}^{k} b_{j i}-b_{i i}=n .
$$

We define $\mathcal{H}_{k, h}^{n}$ to be the set of all matrices $B$ of type $(n, k, h)$. 
Example 3.2. Let $P=(a b)(b c) a_{x} c_{x} \in \operatorname{Sym}_{3}$. Then $\theta(P)=\left(\begin{array}{lll}1 & 1 & 0 \\ 0 & 0 & 1 \\ 0 & 0 & 1\end{array}\right) \in \mathscr{H}_{3,2}^{2}$.

Definition 3.3. Let $\mathbf{H}_{k, h}^{n}$ be the complex vector space generated by the basis elements $[H]$, for $H \in \mathcal{H}_{k, h}^{n}$.

If $H_{1}, H_{2} \in \mathscr{H}_{k, h}^{n}$, then $2\left[H_{1}\right]-3\left[H_{2}\right] \in \mathbf{H}_{k, h}^{n}$. We note that $2\left[H_{1}\right]$ is not the matrix obtained by multiplying the entries of $H_{1}$ by 2 . We can extend the map $\theta$ to a map $\theta: \operatorname{Sym}_{k,(n)^{k}, h} \rightarrow \mathbf{H}_{k, h}^{n}$ by

$$
\theta\left(\sum_{P} c_{P} P\right)=\sum_{P} c_{P}[\theta(P)]
$$

where the sum is over monomials $P$.

The symmetric group $S_{k}$ has a natural action on $\mathscr{H}_{k, h}^{n}$, and thus $\mathbf{H}_{k, h}^{n}$, defined by $\sigma \cdot A=\left(a_{\sigma^{-1}(i) \sigma^{-1}(j)}\right)$, for $A \in \mathscr{H}_{k, h}^{n}$. It follows formally that

Proposition 3.4. The map $\theta: \operatorname{Sym}_{k,(n)^{k}, h} \rightarrow \mathbf{H}_{k, h}^{n}$ is an $S_{k}$-equivariant isomorphism of $\mathbb{C}$-vector spaces.

U-matrices. The following subset of $\mathscr{H}$-matrices will be very useful.

Definition 3.5 ( $U$-matrices). A $U$-matrix is an upper-triangular $\mathscr{H}$-matrix $\left(b_{i j}\right)$ whose diagonal elements form a nonincreasing sequence $b_{11} \geq b_{22} \geq \ldots$. Let $u_{k, h}^{n}$ be the set of all $U$-matrices in $\mathscr{H}_{k, h}^{n}$. Let $\mathbf{U}_{k, h}^{n}$ be the subspace generated by formal complex linear combinations of $u_{k, h}^{n}$-matrices.

Example 3.6. $\left(\begin{array}{lll}3 & 2 & 3 \\ 0 & 2 & 4 \\ 0 & 0 & 1\end{array}\right)$ is a $U$-matrix but $\left(\begin{array}{lll}2 & 1 & 3 \\ 1 & 3 & 3 \\ 1 & 0 & 1\end{array}\right)$ is not.

The $u_{3, h}^{n}$-matrices can be easily parametrized. When $n-h$ is odd, $\cup_{3, h}^{n}=\varnothing$. When $n \equiv h \bmod 2$, we define

$$
\mu_{s, r}=M_{s, r, h, n}=\left(\begin{array}{ccc}
s & r+(n-h) / 2 & h-s-r+(n-h) / 2 \\
0 & h-s-r & s+(n-h) / 2 \\
0 & 0 & r
\end{array}\right),
$$

for integers $r, s$. We usually drop the $h, n$ indices as they are clear from the context. We also define

$$
S_{n, h}=\left\{(s, r) \in \mathbb{Z}^{2} \mid \max \left(0, \frac{1}{2}(h-n)\right) \leq r \leq \frac{1}{3} h, \frac{1}{2}(h-r) \leq s \leq h-2 r\right\} .
$$

Lemma 3.7. Let $n, h$ be nonnegative integers. If $n \equiv h \bmod 2$, then $\mathcal{U}_{3, h}^{n}=\left\{M_{s, r} \mid\right.$ $\left.(s, r) \in S_{n, h}\right\}$. Otherwise $u_{3, h}^{n}=\varnothing$.

Proof. Assume $n \equiv h \bmod 2$. If $(s, r) \in S_{n, h}$, then $s \geq h-s-r \geq r$ and $r+\frac{1}{2}(n-h) \geq$ 0 . Hence $M_{s, r} \in \mathcal{U}_{3, h}^{n}$ for all $(s, r) \in S_{n, h}$. Now suppose $M=\left(b_{i j}\right) \in \mathcal{U}_{3, h}^{n}$. Let $s=b_{11}, r=b_{33}$. The order condition then shows $b_{22}=h-r-s$. Now $s+b_{12}+b_{13}=n, r+b_{13}+b_{23}=n$, and $h-r-s+b_{12}+b_{23}=n$ since $M \in U_{3, h}^{n}$. 
Solving these equations gives the formulas for $b_{12}, b_{13}, b_{23}$ found in $\mu_{s, r}$. Hence, $M=M_{s, r} \in U_{s, r}^{n}$, for some $(s, r) \in S_{n, h}$. The bounds follow from $s \geq h-s-r \geq r \geq 0$ and $b_{12} \geq 0$, proving the first assertion. When $n \neq \equiv h \bmod 2$, the formulas for $b_{12}, b_{21}$ show that these terms cannot be integers if $r, s \in \mathbb{Z}$. Hence $u_{3, h}^{n}=\varnothing$.

Remark 3.8. If $n<h / 3$, then $u_{3, h}^{n}=\varnothing$.

\section{The map $\Delta$}

We,will now construct the maps $\theta, \Delta$ described at the beginning of Section 3 . $\Delta: \mathscr{H}_{3, h}^{n} \rightarrow \mathbf{U}_{3, h}^{n}$ will be a $S_{3}$-invariant map and it will be used to construct a $S_{3}$ equivariant map $(\Delta \circ \theta): \operatorname{Sym}_{3,(n)^{3}, h} \rightarrow \mathbf{U}_{3, h}^{n}$. Recalling that $I \subset \operatorname{Sym}_{3,(n)^{3}, h}$ is the kernel of $\Lambda \circ \chi$, we can then compute the image of $I$ in $\mathbf{U}_{3, h}^{n}$ under $\Delta \circ \theta$. In Section 6 , we will be able to use this result to explicitly classify the covariants in $\mathscr{C}_{3, h}^{n}$ using Corollary 2.7. We begin with the following lemma that follows from the calculation of the $S_{3}$-orbits in $\mathscr{H}_{3, h}^{n}$.

Lemma 4.1. Let $M \in \mathscr{H}_{3, h}^{n}$. Among the matrices $A=\left(a_{i j}\right)$ in the $S_{3}$-orbit of $M$ in $\mathscr{H}_{3, h}^{n}$, there is a unique representative $\widetilde{M}$ satisfying three properties:

(a) $a_{11} \geq a_{22} \geq a_{33}$.

(b) If $a_{11}=a_{22}$, then $\left(a_{12}, a_{13}\right)$ is the largest choice in the lexicographical ordering among the possible choices for $A$.

(c) If $a_{22}=a_{33} \neq a_{11}$, then $\left(a_{23}, a_{13}\right)$ is the largest choice in the lexicographical ordering among the possible A.

Example 4.2. If $M=\left(\begin{array}{lll}1 & 2 & 0 \\ 1 & 2 & 0 \\ 1 & 0 & 4\end{array}\right)$, then $\widetilde{M}=\left(\begin{array}{lll}4 & 0 & 1 \\ 0 & 2 & 1 \\ 0 & 2 & 1\end{array}\right)$. If $M=\left(\begin{array}{lll}2 & 1 & 1 \\ 2 & 2 & 0 \\ 0 & 1 & 4\end{array}\right)$, then $\widetilde{M}=$ $\left(\begin{array}{lll}4 & 1 & 0 \\ 0 & 2 & 2 \\ 1 & 1 & 2\end{array}\right)$.

Definition 4.3. Let $M=\left(m_{i j}\right) \in \mathscr{H}_{3, h}^{n}$. We define $\epsilon_{M}=(-1)^{m_{21}+m_{31}+m_{32}}$,

$$
M^{*}=\left(\begin{array}{ccc}
m_{11} & m_{12}+m_{21} & m_{13}+m_{31} \\
0 & m_{22} & m_{23}+m_{32} \\
0 & 0 & m_{33}
\end{array}\right), \quad \text { and } \quad \bar{M}=\epsilon_{M}\left[M^{*}\right] \in \mathbf{U}_{3, h}^{n} \text {, }
$$

where $[M]$ represents the basis element represented by $M$. We define $\Delta(M)=\overline{\widetilde{M}}$ and extend it to a map $\Delta: \mathbf{H}_{3, h}^{n} \rightarrow \mathbf{U}_{3, h}^{n}$ by

$$
\Delta\left(\sum_{H \in \mathscr{H}_{3, h}^{n}} c_{H}[H]\right)=\sum_{H \in \mathscr{H}_{3, h}^{n}} c_{H} \Delta(H) .
$$

Example 4.4. Let $M=\left(\begin{array}{lll}1 & 1 & 1 \\ 4 & 1 & 3 \\ 3 & 1 & 2\end{array}\right) \in \mathscr{H}_{3,4}^{10}$. Then $\Delta(M)=-\left[M_{2,1}\right] \in \mathbf{U}_{3,4}^{10}$.

Letting $S_{3}$ act trivially on $\mathbf{U}_{3, h}^{n}$, we have: 
Lemma 4.5. $\Delta: \mathbf{H}_{3, h}^{n} \rightarrow \mathbf{U}_{3, h}^{n}$ is a surjective $S_{3}$-equivariant homomorphism of vector spaces.

Proof. If $M \in \mathcal{U}_{3, h}^{n}$, then $\Delta(M)=[M]$. So the subspace in $\mathbf{H}_{3, h}^{n}$ generated by $[M]$, for $M \in \mathcal{U}_{3, h}^{n}$, surjectively maps onto $\mathbf{U}_{3, h}^{n}$.

The composition map $\Delta \circ \theta: \operatorname{Sym}_{3,(n)^{3}, h} \rightarrow \mathbf{U}_{3, h}^{n}$ is a surjective $S_{3}$-equivariant homomorphism of vector spaces. To analyze its kernel, we define the vector spaces

$$
\begin{aligned}
& \mathfrak{a}_{1 ;(n)^{3}, h}:=\mathfrak{a}_{1} \cap \operatorname{Sym}_{3,(n)^{3}, h}, \\
& \mathfrak{a}_{2 ;(n)^{3}, h}:=\mathfrak{a}_{2} \cap \operatorname{Sym}_{3,(n)^{3}, h}, \\
& I_{1}:=\mathfrak{a}_{1 ;(n)^{3}, h}+\left\langle P-\sigma \cdot P \mid P \in \operatorname{Sym}_{3,(n)^{3}, h}\right\rangle \subset \operatorname{Sym}_{3,(n)^{3}, h}, \\
& \mathfrak{g}:=(\Delta \circ \theta)\left(I_{1}\right) \subset \mathbf{U}_{3, h}^{n}, \\
& \mathfrak{h}:=(\Delta \circ \theta)\left(\mathfrak{a}_{2 ;(n)^{3}, h}\right) \subset \mathbf{U}_{3, h}^{n} .
\end{aligned}
$$

We begin by determining $\mathfrak{g}$.

Proposition 4.6. (a) A basis of $\mathfrak{g}$ is given by $\{[B]\}$, with $B=\left(b_{i j}\right) \in \mathcal{U}_{3, h}^{n}$ and

(i) $b_{11}=b_{22} \neq b_{33}$ and $b_{12} \equiv 1 \bmod 2$, or

(ii) $b_{22}=b_{33} \neq b_{11}$ and $b_{23} \equiv 1 \bmod 2$, or

(iii) $b_{11}=b_{22}=b_{33}$ and $b_{12} \equiv 1 \bmod 2$.

(b) The induced map $\overline{\Delta \circ \theta}: \operatorname{Sym}_{3,(n)^{3}, h} / I_{1} \rightarrow \mathbf{U}_{3, h}^{n} / \mathfrak{g}$ is an isomorphism of vector spaces.

Proof of 4.6(a). Since $\Delta \circ \theta$ is $S_{3}$-equivariant, $\mathfrak{g}$ is generated by $(\theta \circ \Delta)\left(\mathfrak{a}_{1 ;(n)^{3}, h}\right)$. Now $\mathfrak{a}_{1 ;(n)^{3}, h}$ is generated by the images $\left(x_{a b}+x_{b a}\right) P$, where $P=\prod_{a \neq b} x_{a b}^{\lambda_{a b}} \prod_{c} x_{c}^{\sigma_{c}}$ is an order $h$ monomial in $\operatorname{Sym}_{3}$ with weight $(n-1, n-1, n),(n-1, n, n-1)$, or $(n, n-1, n-1)$. Let $M=\theta(P)$. By the $S_{3}$-equivariancy, we can assume $M=\left(m_{a b}\right)$ with $m_{11} \geq m_{22} \geq m_{33}$. If $m_{11}>m_{22}>m_{33}$, then $(\Delta \circ \theta)\left(x_{a b} P\right)=-(\Delta \circ \theta)\left(x_{b a} P\right)$ and

$$
(\Delta \circ \theta)\left(\left(x_{a b}+x_{b a}\right) P\right)=0 .
$$

Now suppose $m_{11}=m_{22}>m_{23}$ and $(a, b)=(1,2)$. By working through the various cases, one finds that the only case when $(\Delta \circ \theta)\left(x_{12} P\right)+(\Delta \circ \theta)\left(x_{21} P\right) \neq 0$ occurs when $m_{12}=m_{21}$. In this case, calculation shows $(\Delta \circ \theta)\left(x_{12} P\right)=(\Delta \circ \theta)\left(x_{21} P\right)$ and

$$
(\Delta \circ \theta)\left(\left(x_{12}+x_{21}\right) P\right)=2[B],
$$

where $B=\left(b_{i j}\right)$ is a matrix described in case (i). In particular, $b_{12}=2 m_{12}+1$ is odd. Similar calculations in the other cases establish the rest of (a).

Proof of 4.6(b). By the definition of $\mathfrak{g}$, the map $\overline{\Delta \circ \theta}$ is well-defined. Moreover it is surjective as $\Delta \circ \theta$ is surjective. To show it is injective, we need only 
show that $\operatorname{ker} \Delta \subset \theta\left(I_{1}\right)$ as $\theta$ is an isomorphism. Let $\alpha \in \operatorname{ker} \Delta$ and express $\alpha=\sum_{M \in \mathscr{H}_{3, h}^{n}} c_{M}[M]$. We can rewrite $\alpha$ as $\alpha=\sum_{N \in U_{3, h}^{n}} \alpha_{N}$, where

$$
\alpha_{N}=\sum_{\substack{M \in \mathscr{H}_{3, h}^{n} \\ \Delta(M)= \pm[N]}} c_{M}[M] .
$$

Let $\Delta\left(\alpha_{N}\right)=b_{N}[N]$. Then $0=\Delta(\alpha)=\sum_{N} b_{N}[N]$. Since the elements $[N]$, for $N \in \mathcal{U}_{3, h}^{n}$, form a basis of $\mathbf{U}_{3, h}^{n}$, we have $b_{N}=0, \Delta\left(\alpha_{N}\right)=0$ and $\alpha_{N} \in \operatorname{ker} \Delta$. We now fix $N \in U_{3, h}^{n}$ and show $\alpha_{N} \in \theta\left(I_{1}\right)$.

For each $S_{3}$-orbit $C$ in the set of $M \in \mathscr{H}_{3, n}^{n}$ such that $\Delta(M)= \pm[N]$, let $d_{C}=$ $\sum_{M \in C} c_{M}$. Let $M_{C} \in \mathcal{U}_{3, h}^{n}$ to be the unique representative of $C$ specified by Lemma 4.1. Then

$$
\alpha_{N}=\sum_{\Delta(M)= \pm[N]} c_{M}[M]=\sum_{C} d_{C}\left[M_{C}\right]+\sum_{C} \sum_{M \in C} c_{M}\left([M]-\left[M_{C}\right]\right),
$$

where the sum is over the finite number of orbits $C$. Since $\Delta$ is constant on the orbit $C$, we have

$$
0=\Delta\left(\alpha_{N}\right)=\sum_{C} d_{C} \Delta\left(\left[M_{C}\right]\right) .
$$

Taking the difference of the two equations, we have

$$
\alpha_{N}=\sum_{C} d_{C}\left(\left[M_{C}\right]-\Delta\left(\left[M_{C}\right]\right)\right)+\sum_{C} \sum_{M \in C} c_{M}\left([M]-\left[M_{C}\right]\right) .
$$

The definition of $\Delta$ shows that $\left[M_{C}\right]-\Delta\left(\left[M_{C}\right]\right) \in \theta\left(\mathfrak{a}_{1 ;(n)^{3}, h}\right) \subset \theta\left(I_{1}\right)$. As the second summand is formally in $\theta\left(I_{1}\right), \alpha_{N} \in \theta\left(I_{1}\right)$ and (b) is proved.

Define $\phi: \mathbf{U}_{3, h}^{n} \rightarrow \mathscr{C}_{3, h}^{n}$ by $\phi=\Lambda \circ \chi \circ \theta^{-1}$, where we restrict the domain of the isomorphism $\theta^{-1}: \mathbf{H}_{3, h}^{n} \rightarrow \operatorname{Sym}_{3,(n)^{3}, h}$ to $\mathbf{U}_{3, h}^{n}$.

Corollary 4.7. $\phi$ induces an isomorphism $\mathbf{U}_{3, h}^{n} /(\mathfrak{g}+\mathfrak{h}) \cong \mathscr{C}_{3, h}^{n}$.

Proof. By Corollary 2.7, we have an isomorphism $\Lambda \circ \chi: \operatorname{Sym}_{3,(n)^{3}, h} / I \cong \mathscr{C}_{3, h}^{n}$. Since $k=3$, the syzygies of the first and second kind generate all the syzygies in $\mathfrak{a}$. Hence $I=I_{1}+\mathfrak{a}_{2 ;(n)^{3}, h}$ and $(\Delta \circ \theta)(I)=\mathfrak{g}+\mathfrak{h}$. Using Proposition 4.6(b), it follows that $\Delta \circ \theta$ induces an isomorphism $\operatorname{Sym}_{3,(n)^{3}, h} / I \rightarrow \mathbf{U}_{3, h}^{n} /(\mathfrak{g}+\mathfrak{h})$. Putting these isomorphisms together and noting that $\Delta$ is the identity on $\mathbf{U}_{3, h}^{n}$, gives the claimed isomorphism $\phi$.

\section{Calculation of $\mathfrak{g}+\mathfrak{h}$}

In this section, we explicitly calculate $\mathfrak{g}+\mathfrak{h}$. This calculation is then used in the next section to calculate $\mathscr{C}_{3, h}^{n}$. We begin by calculating $\mathfrak{g}$.

Proposition 5.1. (a) If $n+h \equiv 0 \bmod 4$, then $\mathfrak{g}=\{0\}$. 
(b) If $n+h \equiv 2 \bmod 4$ and $\alpha=\max (0,(h-n) / 2)$, then $\mathfrak{g}$ is generated by

$$
\left\{\left[\mathcal{M}_{h-2 r, r}\right]: \alpha \leq r \leq h / 3\right\} \cup\left\{\left[\mathcal{M}_{(h-r) / 2, r}\right]: \alpha \leq r<h / 3, r \equiv h \quad(\bmod 2)\right\} .
$$

Proof of 5.1(a). Let $n+h=4 t$ for some $t$. Now by Proposition 4.6, $\mathfrak{g}$ is generated by [M], with matrices $M$ of three types: (i), (ii), and (iii). Suppose $M$ has type (i). Then $r=h-2 s$ and

$$
r+\frac{n-h}{2}=h-2 s+\frac{n-h}{2}=\frac{n+h}{2}-2 s=2(t-s)
$$

is even, contradicting the definition of $M$. Similarly, assuming $M$ has types (ii), (iii) leads to contradictions. Hence, $\mathfrak{g}=\{0\}$.

Proof of 5.1(b). By Proposition 4.6, $\mathfrak{g}$ is generated by $\left[\mathcal{M}_{s, r}\right]$, where (i) $s=h-r-s$ or (ii) $h-r-s=r$. Thus $s=(h-r) / 2$ or $h-2 r$. Each of the matrices $M_{h-2 r, r}$, for $\max (0,(h-n) / 2) \leq r \leq h / 3$, and $\mathcal{M}_{(h-r) / 2, r}$, for $0 \leq r \leq h / 3$ and $r \equiv h(\bmod 2)$, is in $u_{3, h}^{n}$. These matrices are distinct except when $s=r=h-r-s, h \equiv 0(\bmod 3)$, and $r=s=h / 3$.

Unfortunately, it is not as simple to describe the generators of $\mathfrak{h}$. Instead, we can determine the generators of $(\mathfrak{g}+\mathfrak{h}) / \mathfrak{g}$. Then by combining them with the generators of $\mathfrak{g}$, we will have a set of generators for $\mathfrak{g}+\mathfrak{h}$. Recalling the definition of $\mu_{s, r}$ in (7), we define

$$
m_{s, r}=\left[\mathcal{M}_{s, r}\right]-\left[\mathcal{M}_{s-1, r}\right]+\left[\mathcal{M}_{s-1, r+1}\right] \in \mathbf{U}_{3, h}^{n},
$$

for nonnegative integers $r, s$ satisfying $r+s \leq h$.

Proposition 5.2. Let $S_{n, h}$ be defined as in (8). Then $(\mathfrak{g}+\mathfrak{h}) / \mathfrak{g}$ is generated by $\Delta\left(m_{s+1, r}\right)$ for all $(s, r) \in S_{n-1, h-1}$.

Before proving Proposition 5.2, we need to establish some lemmas about the functions $\epsilon_{M}, M^{*}, \bar{M}, \widetilde{M}$, and $\Delta(M)$, defined in Definition 4.3.

Lemma 5.3. For $\sigma \in S_{3}, M \in \mathscr{H}_{3, h}^{n}, \epsilon_{\sigma(M)}=\epsilon_{M} \epsilon_{\sigma\left(M^{*}\right)}$.

Proof. Let $M=\left(m_{i j}\right)$. The lemma follows from straightforward calculation for each $\sigma \in S_{3}$. For example, when $\sigma=(12)$, one has

$$
\epsilon_{\sigma(M)}=(-1)^{m_{12}+m_{31}+m_{32}}=(-1)^{m_{21}+m_{31}+m_{32}}(-1)^{m_{12}+m_{21}}=\epsilon_{M} \epsilon_{\sigma\left(M^{*}\right)} .
$$

Lemma 5.4. Suppose we are given $\sigma \in S_{3}, M \in \mathscr{H}_{3, h}^{n}$ with $\sigma(M)^{*}=M^{*}$. Then $\overline{\sigma(M)}=\operatorname{sgn}(\sigma)^{(n+h) / 2} \bar{M}$, where $\operatorname{sgn}$ is the nontrivial homomorphism $\operatorname{sgn}: S_{3} \rightarrow$ $\{ \pm 1\}$.

Proof. We have $\bar{M}=\epsilon_{M} M^{*}$. Then by Lemma 5.3,

$$
\overline{\sigma(M)}=\epsilon_{M} \epsilon_{\sigma\left(M^{*}\right)} \sigma(M)^{*}=\epsilon_{\sigma\left(M^{*}\right)} \bar{M} \text {. }
$$


If $\sigma=1$, then the lemma trivially holds true. If $\sigma \neq 1$, then at least two of the diagonal elements of $M$ are equal. If $\sigma=(12)$, then $m_{11}=m_{22}$. Since $\left(\sigma\left(M^{*}\right)\right)_{21}=$ $(n+h) / 2-m_{11}-m_{22} \equiv(n+h) / 2 \bmod 2, \epsilon_{\sigma\left(M^{*}\right)}=(-1)^{(n+h) / 2}=\operatorname{sgn}(\sigma)^{(n+h) / 2}$ and the lemma is established. Similar calculations establish the lemma for the other choices of $\sigma$.

Lemma 5.5. Assume $n+h$ is even. If $n+h \equiv 0 \bmod 4$ or the diagonal entries of $M \in \mathscr{H}_{3, h}^{n}$ are distinct, then $\Delta(M)=\epsilon_{M} \Delta\left(M^{*}\right)$. Otherwise, $\Delta(M)= \pm \Delta\left(M^{*}\right)$.

Proof. Let $\sigma \in S_{3}$ be such that $\sigma(M)=\widetilde{M}$. We then have

$$
\begin{aligned}
\overline{\sigma\left(M^{*}\right)} & =\epsilon_{\sigma\left(M^{*}\right)} \sigma\left(M^{*}\right)^{*} \\
& =\epsilon_{M} \epsilon_{\sigma(M)} \sigma(M)^{*} \quad(\text { by Lemma 5.3) } \\
& =\epsilon_{M} \Delta(M) .
\end{aligned}
$$

Now if the diagonal elements of $M$ are distinct, $\widetilde{\left(M^{*}\right)}=\sigma\left(M^{*}\right)$ and thus

$$
\Delta\left(M^{*}\right)=\overline{\overline{M^{*}}}=\overline{\sigma\left(M^{*}\right)}=\epsilon_{M} \Delta(M) .
$$

Now suppose the diagonal entries of $M$ are not distinct. Then $\widetilde{\left(M^{*}\right)}=\left(\sigma_{1} \sigma\right)\left(M^{*}\right)$, where $\sigma_{1} \in S_{3}$ has the property that $\left(\sigma_{1}(\sigma(M))\right)^{*}=\sigma(M)^{*}$. By Lemma 5.4,

$$
\Delta\left(M^{*}\right)=\overline{\left(\sigma_{1} \sigma\right)\left(M^{*}\right)}=\operatorname{sgn}\left(\sigma_{1}\right)^{(n+h) / 2} \overline{\sigma(M)}=\operatorname{sgn}\left(\sigma_{1}\right)^{(n+h) / 2} \epsilon_{M} \Delta(M) .
$$

When $n+h \equiv 0 \bmod 4, \operatorname{sgn}\left(\sigma_{1}\right)^{(n+h) / 2}=1$; otherwise, it equals \pm 1 . Hence, the lemma is established.

Proof of Proposition 5.2. By definition, $\mathfrak{h}$ is generated by

$$
(\Delta \circ \theta)\left(\left(x_{i j} x_{k}+x_{j k} x_{i}+x_{k i} x_{j}\right) P\right),
$$

where $P \in \operatorname{Sym}_{3, d-1, h-1}$ is a monomial. Since $\Delta$ is invariant under the action of $S_{3}$ on $\mathcal{H}_{3, h}^{n}$, one can assume that $\theta(P)=\widetilde{\theta(P)}$. Then $\theta(P)^{*}=\mu_{s, r}$, with $(s, r) \in$ $S_{n-1, h-1}$. It is also enough to consider the cases $(i, j, k)=(1,2,3),(1,3,2)$. Assume $(i, j, k)=(1,2,3)$. Then $\theta\left(x_{23} x_{1} P\right)^{*}=\mu_{s+1, r}$ and $\left(\theta\left(\widetilde{x_{23} x_{1} P}\right)\right)^{*}=M_{s+1, r}$ by the assumption on $P$, so

$$
(\Delta \circ \theta)\left(x_{23} x_{1} P\right)=\epsilon_{P} \Delta\left(M_{s+1, r}\right) .
$$

When $n+h \equiv 0 \bmod 4$, by Lemma 5.5, $\theta\left(x_{31} x_{2} P\right)^{*}=\mu_{s, r}, \theta\left(x_{12} x_{3} P\right)^{*}=M_{s, r+1}$, and thus

$$
(\Delta \circ \theta)\left(x_{31} x_{2} P\right)=-\epsilon_{P} \Delta\left(M_{s, r}\right), \quad(\Delta \circ \theta)\left(x_{12} x_{3} P\right)=\epsilon_{P} \Delta\left(M_{s, r+1}\right) .
$$

Thus (9) equals $\epsilon_{P} \Delta\left(m_{s+1, r}\right)$. When $(i, j, k)=(1,3,2)$, one gets $-\epsilon_{P} \Delta\left(m_{s+1, r}\right)$, proving the lemma when $n+h \equiv 0 \bmod 4$. Now suppose $n+h \equiv 2 \bmod 4$ and consider $x_{31} x_{2} P$. If the diagonal elements of $\theta\left(x_{31} x_{2} P\right)$ are distinct, we have 
$(\Delta \circ \theta)\left(x_{31} x_{2} P\right)=-\epsilon_{P} \Delta\left(M_{s, r}\right)$ by Lemma 5.5. If they are not distinct, then $(\Delta \circ \theta)\left(x_{31} x_{2} P\right)=-\epsilon_{P} \Delta\left(M_{s, r}\right)$ in $\mathfrak{g} /(\mathfrak{g}+\mathfrak{h})$ as both sides are 0 by Proposition 5.1. The same logic shows $(\Delta \circ \theta)\left(x_{12} x_{3} P\right)=\epsilon_{P} \Delta\left(M_{s, r+1}\right)$, establishing (9) and proving the proposition when $n+h \equiv 2 \bmod 4$.

To make the generators of $(\mathfrak{g}+\mathfrak{h}) / \mathfrak{g}$ explicit, we define the following elements of $\mathbf{H}_{3, h}^{n}$ :

$$
\begin{aligned}
& n_{s, r}=\left[M_{s, r}\right]-\left(1+(-1)^{(n+h) / 2}\right)\left[\mathcal{M}_{s-1, r}\right], \\
& p_{s, r}=\left(1+(-1)^{(n+h) / 2}\right)\left[\mathcal{M}_{s, r}\right]+\left[\mathcal{M}_{s-1, r+1}\right],
\end{aligned}
$$

for integers $s, r \geq 0$. In general, $n_{s, r}, p_{s, r}$ will not be elements of $\mathbf{U}_{3, h}^{n}$.

Proposition 5.6. $(\mathfrak{g}+\mathfrak{h}) / \mathfrak{g}$ is generated by the following elements of $\mathbf{U}_{3, h}^{n}$ :

(a) $m_{s, r}$, where $\max (0,(h-n) / 2) \leq r \leq(h-4) / 3,(h-r) / 2+1 \leq s \leq h-2 r-1$.

(b) $n_{h-2 r, r}$, where $\max (0,(h-n) / 2) \leq r \leq(h-2) / 3$.

(c) $p_{(h-r+1) / 2, r}$, where $\max (0,(h-n) / 2) \leq r \leq(h-3) / 3$ and $r \equiv h+1 \bmod 2$.

(d) $\left[\mathcal{M}_{(h+2) / 3,(h-1) / 3}\right]$, if $h \equiv 1 \bmod 3$.

Proof. By Proposition 5.2, h is generated by $\Delta\left(m_{s, r}\right)$, where $(s-1, r) \in S_{n-1, h-1}$. It follows immediately that $(s, r) \in S_{n, h}$ and $\mathcal{M}_{s, r} \in \mathcal{U}_{3, h}^{n}$. However, the terms $M_{s-1, r}, M_{s-1, r+1}$ might not be in $\mathcal{U}_{3, h}^{n}$, so we need to do a case-by-case analysis. Since $(s-1, r) \in S_{n-1, h-1}$, we have $s-1 \geq h-s-r \geq r$. We separately analyze the cases when we have equality or strict inequality.

Case (a): Suppose $s-1>h-s-r>r$. Then $(s-1, r),(s-1, r+1) \in S_{n, h}$, $\overline{\text { and } \mathcal{M}_{s-1, r}}, \mathcal{M}_{s-1, r+1}$ in $\mathcal{U}_{3, h}^{n}$. Thus $\Delta\left(m_{s, r}\right)=m_{s, r}$. We now prove the claimed inequalities for $s, r$. From the assumptions, we have $2 s \geq h-r+2$ and $h \geq$ $s+2 r+1$, giving the claimed conditions on $s$. Combining these equations, we obtain $h \geq(h-r) / 2+2 r+2$ and $h \geq 3 r+4$. Thus $r \leq(h-4) / 3$. The lower bound on $r$ follows from $(s-1, r) \in S_{n-1, h-1}$. Conversely, if $s, r$ satisfies the bounds in (a), then one can show that $s-1>h-s-r>r$ and $(s-1, r) \in S_{n-1, h-1}$.

Case (b): Suppose $s-1>h-s-r$ and $h-s-r=r$. Then $(s-1, r)$ is an element of $S_{n, h}$ but $(s-1, r+1)$ is not. Hence $\mathcal{M}_{s-1, r} \in \mathcal{U}_{3, h}^{n}$, but $M_{s-1, r+1} \notin \mathcal{U}_{3, h}^{n}$. Since $s=h-2 r$, we have

$$
\Delta\left(M_{s-1, r+1}\right)=(-1)^{s-1+(n-h) / 2}\left[\mathcal{M}_{s-1, r}\right]=(-1)^{1+h+(n-h) / 2}\left[\mathcal{M}_{s-1, r}\right],
$$

and $\Delta\left(m_{s, r}\right)=n_{s, r} \in \mathbf{U}_{3, h}^{n}$. To establish the bounds, we see that $s-1 \geq r+1$. Then

$$
3 r+1=r+r+(r+1) \leq r+(h-s-r)+(s-1)=h-1
$$

and $r \leq(h-2) / 3$. Conversely, if $r$ satisfies the stated bounds in (b), and $s=h-2 r$, one can show that $(s-1, r) \in S_{n-1, h-1}$ and $s-1>h-s-r=r$. 
Case (c): Suppose $s-1=h-s-r$ and $h-s-r>r$. Then $(s-1, r+1)$ is an element of $S_{n, h}$, but $(s-1, r)$ is not. Then $M_{s-1, r+1} \in \mathcal{U}_{3, h}^{n}$, but $M_{s-1, r} \notin \mathcal{U}_{3, h}^{n}$. As $r=h+1-2 s, r$ and $h$ have different parities. Then

$$
\Delta\left(M_{s-1, r}\right)=(-1)^{r+(n-h) / 2}\left[M_{s, r}\right]=(-1)^{1+h+(n-h) / 2}\left[M_{s, r}\right]
$$

and $\Delta\left(m_{s, r}\right)=\left[1+(-1)^{(n+h) / 2}\right]\left[\mathcal{M}_{s, r}\right]+\left[\mathcal{M}_{s-1, r+1}\right]=p_{s, r}$. Now $h-2 r-1 \geq a$, so $b=h+1-2 s \geq h+1-2(h-2 r-1)$ and $r \leq(h-3) / 3$. Conversely, when the stated conditions on $r$ hold, one can show that $(s, r) \in S_{n-1, h-1}$, with $a=(h-r+1) / 2$, and $s-1=h-s-r>b$.

Case (d): Suppose $s-1=h-s-r=r$. Then $h-1=3 r$ and $h \equiv 1 \bmod 3$. In this case, neither $(s-1, r)$ nor $(s-1, r+1)$ is in $S_{n, h}$. Then

$$
\Delta\left(M_{s-1, r}\right)=(-1)^{r+(n-h) / 2}\left[\mathcal{M}_{s, r}\right]=-(-1)^{(n+h) / 2}\left[\mathcal{M}_{s, r}\right],
$$

and $\Delta\left(M_{s-1, r+1}\right)=(-1)^{(n+h) / 2}\left[M_{s, r}\right]$. Thus

$$
\Delta\left(m_{s, r}\right)=\Delta\left(\left[\mathcal{M}_{s, r}\right]-\left[\mathcal{M}_{s-1, r}\right]+\left[\mathcal{M}_{s-1, r+1}\right]\right)=\left(1+2(-1)^{(n+h) / 2}\right)\left[\mathcal{M}_{s, r}\right] .
$$

Regardless of whether $n+h \equiv 0,2 \bmod 4,\left[\mathcal{M}_{s, r}\right] \in \mathfrak{h}$. Since $s=(h+2) / 3$, $r=(h-1) / 3$, we obtain the result in (d) in the proposition.

\section{Calculation of degree-three covariants}

In this section, we determine an explicit basis for $\mathscr{C}_{3, h}^{n}$ in Theorem 6.1 and derive the formulas for $\operatorname{dim} \mathscr{C}_{3, h}^{n}$ in Table 1 as a corollary. We establish these results by combining the calculation of $\mathfrak{g}+\mathfrak{h}$ from the previous section with Corollary 4.7 to calculate $\mathscr{C}_{3, h}^{n}$. To simplify the statement of the theorem, we use the map $\phi: \mathbf{U}_{3, h}^{n} \rightarrow$ $\mathscr{C}_{3, h}^{n}$ defined before Corollary 4.7. $\phi$ has the property that if $M=\left(m_{i j}\right) \in \mathcal{U}_{3, h}^{n}$, then

$$
\phi([M])=\prod_{a \neq b}(a b)^{m_{a b}} \prod_{c} c_{x}^{m_{c c}},
$$

where we use the classical symbolic notation for $\mathscr{C}_{3, h}^{n}$ (see page 516).

Theorem 6.1. Let $n, h$ have the same parity. Then a basis of $\mathscr{C}_{3, h}^{n}$ is given as follows.

(a) If $n+h \equiv 1 \bmod 2$, then $\mathscr{C}_{3, h}^{n}=\{0\}$.

(b) When $n+h \equiv 0 \bmod 4$, the elements $\phi\left(\left[\mu_{(h-r) / 2, r}\right]\right)$, where $\max \left(0, \frac{h-n}{2}\right) \leq$ $r \leq h / 3$ and $r \equiv h \bmod 2$, form a basis.

(c) If $n+h \equiv 2 \bmod 4$, the elements $\phi\left(\left[\mu_{(h-r+1) / 2, r}\right]\right)$, where $\max \left(0, \frac{h-n}{2}\right) \leq r<$ $(h-1) / 3$ and $r \equiv h+1 \bmod 2$ form a basis. 
Example 6.2. When $n=10, h=12$, Theorem 6.1(c) states that $\left\{\phi\left(\left[\mathcal{M}_{6,1}\right]\right)\right.$, $\left.\phi\left(\left[M_{5,3}\right]\right)\right\}$ is a basis of $\mathscr{C}_{3,12}^{10}$. Using the classical symbolic notation (compare Example 2.5), these covariants are $(a c)^{4}(b c)^{5} a_{x}^{6} b_{x}^{5} c_{x}$ and $(a b)^{2}(a c)^{3}(b c)^{5} a_{x}^{5} b_{x}^{4} c_{x}^{3}$. Proof of 6.1. By Corollary 4.7,

$$
\mathscr{C}_{3, h}^{n} \cong \mathbf{U}_{3, h}^{n} /(\mathfrak{g}+\mathfrak{h}) \cong\left(\mathbf{U}_{3, h}^{n} / \mathfrak{g}\right) /(\mathfrak{g}+\mathfrak{h} / \mathfrak{g}) .
$$

By Lemma 3.7, (a) is established. We now consider cases (b) and (c). By the same lemma, the set

$$
\left\{\left[\mathcal{M}_{s, r}\right] \mid(s, r) \in S_{n, h}\right\}
$$

is a basis for $\mathbf{U}_{3, h}^{n}$. We define an order on the basis elements $\mathcal{M}_{s, r}$ by defining $\left[\mathcal{M}_{s, r}\right] \geq\left[\mathcal{M}_{s^{\prime}, r^{\prime}}\right]$ if $(s, h-r-s, r) \geq\left(s^{\prime}, h-r^{\prime}, s^{\prime}, r^{\prime}\right)$ in the lexicographic order. We will prove the proposition by ordering the generators of $\mathfrak{g}+\mathfrak{h}$, from largest to smallest, by their largest terms (which will be distinct). Let $a=\operatorname{dim} \mathbf{U}_{3, h}^{n}$ and $b=\operatorname{dim}(\mathfrak{g}+\mathfrak{h})$. By expressing the generators of $\mathfrak{g}+\mathfrak{h}$ in terms of the $\left[\mathcal{M}_{s, r}\right]$, we obtain a $b \times a$ upper-triangular matrix $\mathscr{A}$ of relations. A basis of the quotient space $\mathbf{U}_{3, h}^{n} /(\mathfrak{g}+\mathfrak{h})$ is then given by the cosets of $\left[\mathcal{M}_{s, r}\right]$, for $(s, r)$ corresponding to nonpivot columns of $\mathscr{A}$. We now separately analyze the details of parts (b) and (c).

(b) When $n+h \equiv 0 \bmod 4, \mathfrak{g}=0$ and we are reduced to calculating the quotient space $\mathbf{U}_{3, h}^{n} / \mathfrak{h}$. Proposition 5.6 gives a basis for the vector space $\mathfrak{h}$ and the leading terms of each of the $m_{s, r}, n_{s, r}, p_{s, r}$ specified in Proposition 5.6 are $\left[\mu_{s, r}\right]$. We note that by the proof of Proposition 5.6, the specified pairs $(s, r)$ are distinct and comprise all $(s, r) \in S_{n, h}$ with $(s-1, r) \in S_{n-1, h-1}$. Hence the generators of $\mathbf{U}_{3, h}^{n} / \mathfrak{h}$ are the $\left[\mathcal{M}_{s, r}\right]$ for those pairs $(s, r) \in S_{n, h}$ with $(s-1, r) \notin S_{n-1, h-1}$. The definition of $S_{n, h}$ shows that such a pair $(s, r)$ occurs precisely when $3 \mid h$ and $r=h / 3$ or when $h-r$ is even and $s=(h-r) / 2$. In the first case, let $h=3 t$. Then $r=t$ and the condition on $s$ shows $s=t$. As such, this situation is a subcase of the second case. $\mathscr{C}_{3, h}^{n}$ is thus generated by the $\left[\mathcal{M}_{s, r}\right]$, for $(s, r)$ in the second case, and this is what the proposition states.

(c) By Propositions 5.1 and 5.6, $\mathfrak{g}+\mathfrak{h}$ is generated by $\left[\mathcal{M}_{s, r}\right]$ where $2 s=h-r$ or $2 r=h-s$, and by $m_{s, r}, n_{h-2 r, r}, p_{(h-r+1) / 2, r}$. Since $n+h \equiv 2 \bmod 4, n_{h-2 r, r}=$ $\left[\mathcal{M}_{h-2 r, r}\right]$, and $p_{(h-r+1) / 2, r}=\left[\mathcal{M}_{(h-r-1) / 2, r+1}\right]$ and both of them are included in the former set. The leading term of $m_{s, r}$ is $\left[\mathcal{M}_{s, r}\right]$ and the corresponding $(s, r)$ specified in Proposition 5.6 are all those pairs $(s, r) \in S_{n, h}$ with $s-1>h-s-r>r$. Since $(s, r) \in S_{n, h}$ implies $s \geq h-s-r \geq r$, the generators of $\mathbf{U}_{3, h}^{n} /(\mathfrak{g}+\mathfrak{h})$ are the $\left[\mathcal{M}_{s, r}\right]$ for which $(s, r) \in S_{n, h}, s-1=h-s-r$, and $h-s-r>r$, which is what the proposition claims. 
When $n \geq h$ :

\begin{tabular}{c|c|c} 
& $n+h \equiv 0 \bmod 4$ & $n+h \equiv 2 \bmod 4$ \\
\hline$h$ even & {$\left[\frac{h}{6}\right]+1$} & {$\left[\frac{h}{6}\right]$} \\
\hline$h$ odd & {$\left[\frac{h+3}{6}\right]$} & {$\left[\frac{h+3}{6}\right]$} \\
\hline
\end{tabular}

When $\frac{1}{3} h \leq n \leq h$ :

\begin{tabular}{c|c|c}
$h \bmod 3$ & $n+h \equiv 0(\bmod 4)$ & $n+h \equiv 2(\bmod 4)$ \\
\hline 0 & {$\left[\frac{3 n-h}{12}\right]+1$} & {$\left[\frac{3 n-h-2}{12}\right]+1$} \\
\hline 1 & {$\left[\frac{3 n-h}{12}\right]+1$} & {$\left[\frac{3 n-h-2}{12}\right]$} \\
\hline 2 & {$\left[\frac{3 n-h}{12}\right]+1$} & {$\left[\frac{3 n-h-2}{12}\right]+1$} \\
\hline
\end{tabular}

Table 1. Formulas for $\operatorname{dim} \mathscr{C}_{3, h}^{n}$.

Corollary 6.3. The dimension of the vector space $\mathscr{C}_{3, h}^{n}$ is given by the formulas in Table 1 when $n, h$ have the same parity and $n \geq h / 3$. Otherwise $\operatorname{dim} \mathscr{C}_{3, h}^{n}=0$.

Corollary 6.3 can also be derived via an explicit calculation using the classical Cayley-Sylvester formula for calculating $\operatorname{dim} \mathscr{C}_{d, h}^{n}$ [Sturmfels 2008, p. 153]. We note that the case $n=h$ appears twice in Table 1 with seemingly different formulas, but calculation shows that the formulas agree.

Theorem 6.1 parametrizes all the covariants in $\mathscr{C}_{3, h}^{n}$. We say $f \in \mathscr{C}_{3, h}^{n}$ is a reducible covariant if $f$ can be written as a linear combination of products $g h$ of covariants $g, h$ with degree less than $3, \operatorname{deg} g+\operatorname{deg} h=3$, and ord $g+\operatorname{ord} h=h$ Let $\operatorname{Red}_{3, h}^{n}$ be the subspace of $\mathscr{C}_{3, h}^{n}$ generated by the reducible covariants. An irreducible covariant $f \in \mathscr{C}_{3, h}^{n}$ is a covariant that is not reducible.

By Example 2.8, the only nonzero covariants of degree one are multiples of $(\Lambda \circ \chi)\left(x_{1}^{n}\right)$. By Example 2.9, the only nonzero degree-two covariants of order $h$ occur when $0 \leq h \leq 2 n$ and $h \equiv 2 n \bmod 4$. In this case, they are given by multiples of $(\Lambda \circ \chi)\left(x_{12}^{n-(h / 2)} x_{1}^{h / 2} x_{2}^{h / 2}\right)$. Thus, $\operatorname{Red}_{3, h}^{n}$ is a one-dimensional space precisely when $h \geq n$ and $h+n \equiv 0 \bmod 4$, in which case it is generated by $\mathcal{M}_{n,(h-n) / 2}$. Otherwise $\operatorname{Red}_{3, h}^{n}=\{0\}$.

Corollary 6.4. If $h<n$ or $h+n \not \equiv 0 \bmod 4$, then $\operatorname{Red}_{3, h}^{n}=\{0\}$ and $\mathscr{C}_{3, h}^{n}$ contains no reducible covariants. If $h \geq n$ and $h+n \equiv 0 \bmod 4$, then the covariants $\phi\left(\left[\mathcal{M}_{(h-r) / 2, r}\right]\right)$, where $r \equiv h \bmod 2$ and $(h-n) / 2<r \leq h / 3$, form a basis for the subspace of $\mathscr{C}_{3, h}^{n}$ complementary to $\operatorname{Red}_{3, h}^{n}$.

Proof. Only the second part remains to be shown. In this case, $\operatorname{Red}_{3, h}^{n}$ is generated by $\left[\mathcal{M}_{n,(h-n) / 2}\right]$. We can assume $h \leq 3 n$ as otherwise there are no nonzero 
covariants. If $h=3 n$, then $(n,(h-n) / 2)=(n, n)$, and $\left[\mathcal{M}_{n,(h-n) / 2}\right]$ is one of the basis elements of $\mathscr{C}_{3, h}^{n}$ given by Theorem 6.1. By deleting this basis element, the remaining basis elements give a basis for the subspace of $\mathscr{C}_{3, h}^{n}$ complementary to $\operatorname{Red}_{3, h}^{n}$. Now let $h=3 n-4 k$, where $k \geq 1$. By using a syzygy of the second type, we have

$$
\left[\mathcal{M}_{n,(h-n) / 2}\right]=\left[\mathcal{M}_{n, n-2 k}\right]=2\left[\mathcal{M}_{n-1, n-2 k}\right]
$$

in $\mathbf{U}_{3, h}^{n} /(\mathfrak{g}+\mathfrak{h})$. If $k=1,(n-1, n-2 k)=((h-r) / 2, r)$ for $n=h-2 k$, and $\left[\mathcal{M}_{n,(h-n) / 2}\right]=\left[\mathcal{M}_{(h-r) / 2, r}\right]$ is again one of the basis elements of $\mathscr{C}_{3, h}^{n}$ given by Theorem 6.1. By excluding this basis element, we obtain the desired basis for the complementary subspace. Now suppose $k>1$. Then by applying the second syzygy $k-1$ additional times, we obtain in $\mathbf{U}_{3, h}^{n} /(\mathfrak{g}+\mathfrak{h})$

$$
\left[\mathcal{M}_{n, n-2 k}\right]=2\left[\mathcal{M}_{n-k, n-2 k}\right]+\sum_{r=1}^{k-1} c_{r}\left[\mathcal{M}_{n-k, n-2 k+r}\right],
$$

for some constants $c_{r}$. Now by the ordering introduced in the proof of Theorem 6.1, $\left[\mathcal{M}_{n-k, n-2 k}\right]>\left[\mathcal{M}_{n-k, n-2 k+r}\right]$ for $1 \leq r \leq k-1$, and thus each $\left[\mathcal{M}_{n-k, n-2 k+r}\right]$ in the summand can be expressed as a linear combination of $\left[\mathcal{M}_{s, r}\right]$ given by Theorem 6.1 with $\left[\mathcal{M}_{s, r}\right]<\left[\mathcal{M}_{n-k, n-2 k}\right]$. Let $\mathscr{I}$ be the space generated by the $\left[\mathcal{M}_{(h-r) / 2, r}\right]$ specified in the Corollary. Each $\left[\mathcal{M}_{n-k, n-2 k+r}\right]$ in the summand can then be expressed as a linear combination of elements in $\mathscr{I}$. Thus the space generated by $\left[\mathcal{M}_{n, n-2 k}\right]$ and $\mathscr{T}$ is also the space generated by $\left[M_{n-k, n-2 k}\right]$ and $\mathscr{T}$. Since $(n-k, n-2 k)=\left(\frac{h-r}{2}, r\right)$ for $r=n-2 k$, this is $\mathscr{C}_{3, h}^{n}$, by Theorem 6.1. Thus $\mathscr{I}$ is the subspace of $\mathscr{C}_{3, h}^{n}$ complementary to $\operatorname{Red}_{3, h}^{n}$.

Historical remark. We would like to note a correction to a claim in Hilbert's fundamental book on covariants [1993]. First, we define the weight $w$ of a covariant of degree $d$, order $h$, and degree- $n$ form to be $w=(d n-h) / 2$. When $d=3$,

$$
w=(3 n-h) / 2=2 n-(n+h) / 2 .
$$

On [Hilbert 1993, p. 62], there appears the statement:

"Regarding the covariants of degree three, they all have odd weight $p=$ $2 \pi+1$ and are those which occur in the following expression, where $p=3,5,7, \ldots, n$, respectively $n-1: "$

Hilbert then gives an explicit formula for a covariant $f_{p}$ of weight $p$. In total, the claim is that all degree-three covariants have odd weight and that there is exactly one nonreducible covariant of each odd weight $3 \leq p \leq n$. It is clear, both from the Cayley-Sylvester formula and from Theorem 6.1, that this is incorrect. From Theorem 6.1, one sees that in general there are many covariants with a given even 
weight when $n+h \equiv 0 \bmod 4$. Similarly with $n+h \equiv 2 \bmod 4$, there are generally multiple covariants of a given odd weight.

Comparison with result of Kraft and Weyman. Kraft and Weyman [1999, Theorem 6.7] establish a generating set for the covariants in $\mathscr{C}_{3, h}^{n}$ with similarities to our Theorem 6.1. We now briefly discuss the differences between these results. Using the classical symbolic notation (see page 516), let

$$
P=(a b)^{\alpha}(b c)^{\beta}(c a)^{\gamma} a_{x}^{n-\alpha-\gamma} b_{x}^{n-\alpha-\beta} c_{x}^{n-\beta-\gamma} \in \mathscr{C}_{3, h}^{n}
$$

be a covariant of order $h=3 n-2 m$, where $m=\alpha+\beta+\gamma$. We assume $n \geq$ $\max (\alpha+\beta, \alpha+\gamma, \beta+\gamma)$. We define cat $P:=\max (\alpha, \beta, \gamma)$. Kraft and Weyman prove:

Theorem 6.5 (Kraft and Weyman's abc-Theorem). Assume $n, h \geq 0$.

(a) If $n \leq h$, then $P$ is a linear combination of covariants

$$
Q=(a b)^{\mu}(b c)^{\eta} a_{x}^{n-\mu} b_{x}^{n-\mu-\eta} c_{x}^{n-\eta}
$$

with $\mu+\eta=m, \mu \geq 2 \eta$ and $\mu \geq$ cat $P$.

(b) If $h=n$, then $P$ belongs to the ideal $\Phi \subset P\left(V_{n}\right)$ generated by all covariants of degree $k \leq 2$ and order $h \leq \frac{3}{4} n$.

(c) If $n \geq h$, then $P$ belongs to the ideal $\mathscr{F} \subset P\left(V_{n}\right)$ generated by all covariants with degree $k \leq 3$ and order $h \leq \frac{3}{4} n$.

Since $\mathscr{C}_{3, h}^{n}$ is generated by $P$, as $\alpha, \beta, \gamma$ vary, part (a) of Theorem 6.5 gives a spanning set for the vector space $\mathscr{C}_{3, h}^{n}$ when $n \leq h$. However, this spanning set is almost always linearly dependent and doesn't give a basis for $\mathscr{C}_{3, h}^{n}$. For example, when $n=10, h=12$, part (a) shows that $\mathscr{C}_{3,12}^{10}$ is generated by the three symbolic monomials $Q$ corresponding to $(\mu, \eta)=(6,3),(7,2)$, and $(8,1)$ (the covariant corresponding to $(\mu, \eta)=(9,0)$ is zero). However, $\operatorname{dim} \mathscr{C}_{3,12}^{10}=2$ by Table 1, and Example 6.2 shows that the two monomials $(a c)^{4}(b c)^{5} a_{x}^{6} b_{x}^{5} c_{x}$ and $(a b)^{2}(a c)^{3}(b c)^{5} a_{x}^{5} b_{x}^{4} c_{x}^{3}$ form a basis for $\mathscr{C}_{3,12}^{10}$.

Similarly, parts (b), (c) of Theorem 6.5 show that the vector space of covariants $\mathscr{C}_{3, h}^{n}$ is contained in the respective ideals $\mathscr{\Phi}, \mathscr{F}$ of the ring $P\left(V_{n}\right)$, but do not establish a basis for $\mathscr{C}_{3, h}^{n}$. To see the difference, we consider the case when $n=h=3$. Then part (b) states that $\mathscr{C}_{3,3}^{3}$ is contained in the ideal $\mathscr{I}$ of $P\left(V_{3}\right)$ (a ring containing both covariant and noncovariant functions) generated by reducible covariants. However, by Corollary 6.4, we know that $\operatorname{Red}_{3,3}^{3}=\{0\}$ and $\mathscr{C}_{3,3}^{3}$ is generated as a vector space by a single irreducible covariant. 


\section{Acknowledgements}

It is a pleasure to thank The College of New Jersey for its support of this work through its Summer Undergraduate Research Program. We would like to thank both J. Hatley for his computational help that aided our calculations, and the referee for many helpful suggestions.

\section{References}

[Bedratyuk 2009] L. Bedratyuk, "A complete minimal system of covariants for the binary form of degree 7”, J. Symbolic Comput. 44:2 (2009), 211-220. MR 2009j:13006 Zbl 05494972

[Bedratyuk and Bedratyuk 2008] L. Bedratyuk and S. Bedratyuk, "A complete system of covariants for the binary form of the eighth degree", Mat. Visn. Nauk. Tov. Im. Shevchenka 5 (2008), 11-22. In Ukrainian; English version in http://www.arxiv.org/abs/math/0612113. Zbl 05592395

[Dolgachev 2003] I. Dolgachev, Lectures on invariant theory, London Mathematical Society Lecture Note Series 296, Cambridge University Press, 2003. MR 2004g:14051 Zbl 1023.13006

[Gordan 1868] P. Gordan, "Beweis, dass jede Covariante und Invariante einer binären Form eine ganze Funktion mit numerischen Coefficienten einer endlichen Anzahl solcher Formen ist", J. Reine und Angewandte Mathematik 69 (1868), 323-354. JFM 01.0060.01

[Hilbert 1890] D. Hilbert, "Ueber die Theorie der algebraischen Formen”, Math. Ann. 36:4 (1890), 473-534. MR 1510634

[Hilbert 1893] D. Hilbert, "Ueber die vollen Invariantensysteme”, Math. Ann. 42:3 (1893), 313-373. MR 1510781 JFM 25.0173.01

[Hilbert 1993] D. Hilbert, Theory of algebraic invariants, Cambridge University Press, 1993. MR 97j.01049 Zbl 0801.13001

[Howe 1994] R. Howe, "The invariants of degree up to 6 of all $n$-ary $m$-ics", pp. 335-348 in Lie theory and geometry, edited by J.-L. Brylinski et al., Progr. Math. 123, Birkhäuser, Boston, MA, 1994. MR 96f:13007 Zbl 0901.15018

[Kraft and Weyman 1999] H. Kraft and J. Weyman, "Degree bounds for invariants and covariants of binary forms", preprint, 1999, Available at www.math.unibas.ch/ kraft/Papers/KWJordan.pdf.

[Kung and Rota 1984] J. P. S. Kung and G.-C. Rota, "The invariant theory of binary forms", Bull. Amer. Math. Soc. (N.S.) 10:1 (1984), 27-85. MR 85g:05002 Zbl 0577.15020

[Olver 1999] P. J. Olver, Classical invariant theory, London Mathematical Society Student Texts 44, Cambridge University Press, 1999. MR 2001g:13009 Zbl 0971.13004

[Procesi 2007] C. Procesi, Lie groups, Springer, New York, 2007. MR 2007j:22016 Zbl 1154.22001

[Sturmfels 2008] B. Sturmfels, Algorithms in invariant theory, 2nd ed., Springer, Vienna, 2008. MR 94m:13004 Zbl 1154.13003

Received: 2008-10-16 Revised: 2009-12-08 Accepted: 2009-12-21

hagedorn@tcnj.edu

Department of Mathematics and Statistics,

The College of New Jersey, P.O. Box 7718,

Ewing, NJ 08628-0718, United States

glenmatthewwilson@gmail.com Department of Mathematics and Statistics,

The College of New Jersey, P.O. Box 7718,

Ewing, NJ 08628-0718, United States 